\title{
Experimental Study of Rayleigh-Bénard Convection in the Presence of Rotation
}

\author{
Sridhar Balasubramanian and Robert E. Ecke
}

\begin{abstract}
Laboratory experiments were conducted to study heat transport mechanisms in Rayleigh-Bénard convection subjected to rotation along the vertical axis. The parameter range for this study was Rayleigh numbers, $R a=1 \times 10^{7}-2 \times 10^{8}$, and Taylor numbers, $T a=0-1.89 \times 10^{10}$. The working fluid was water with a Prandtl number $P r=6$. Heat transport was measured for varying rotation rates for a fixed set of Rayleigh numbers. The Nusselt number, $\mathrm{Nu}$, plotted as a function of $\mathrm{Ra}$ shows dependence on rotation rates. The vertical heat transport measurements show presence of coherent structures in the presence of rotation. These structures also show some periodicity for a range of rotation rates. The data is roughly consistent with an assumption that the enhancement of heat transport owing to rotation is proportional to the vortical structures penetrating the boundary layer. The vertical heat transport measurements assess the role of Ekman pumping in controlling heat transport in different regimes of Rayleigh and Taylor numbers.
\end{abstract}

Index Terms-Turbulent convection, heat transport, coherent structures, ekman pumping.

\section{INTRODUCTION}

The Rayleigh-Bénard type turbulent thermal convection arising due to the temperature differences is a common occurrence in many geophysical and astrophysical flows [1], [2]. Some examples are heat exchanger systems, oceanic deep convection, convection in stars and giant planets. The presence of various factors such as rotation, stratification or double diffusion adds complexity to the study of such fluid flows. The effects of stratification and double diffusion has been studied vastly, however the rotation effects arising due to the Coriolis parameter has been ignored in most of the thermal convection studies [3], [4]. One of the reasons could be attributed to the fact that widespread belief among atmospheric scientists that the Earth's Coriolis forces are not important in determining the turbulence structure of the planetary boundary layer. However, recent studies have shown that rotation plays a very important role in the atmosphere-ocean dynamics, and can't be ignored [5]-[7]. The main focus of this study is to understand the effects of rotation on turbulent thermal convection, which is critical in

Manuscript received November 22, 2012; revised February 3, 2013. This research work was supported mainly by the LDRD-DR program at Los Alamos National Laboratory and in part by the institute funds from Indian Institute of Technology, Bombay.

S. Balasubramanian was with Los Alamos National Laboratory, Los Alamos, NM, 87545, USA. He is now with the Department of Mechanical Engineering, Indian Institute of Technology, Bombay, MH, 400076, India (e-mail: sridharb@iitb.ac.in).

R. E. Ecke is with Center for Non Linear Studies, Los Alamos National Laboratory, Los Alamos, NM, 87545, USA (e-mail: ecke@lanl.gov). determining the nature of the fluid motions in ocean and atmosphere. Over the past few years, research papers have reported that rotation in the form of Coriolis effect plays an important role in understanding the origins of heat transport scaling in turbulent convection [8]-[10]. The effects of rotation on the thermal convective flows arose from the pioneering theoretical work of Chandrasekhar [1], where it was reported that the rotation modifies the Rayleigh-Bénard type convective stability, by suppressing the onset of convection. Since, then lot of experimental and numerical studies has been done on this topic. Experimental measurements of heat transport in rotating convection include the seminal work of Rossby [3] and later studies that also had qualitative flow visualization [9], [11], [12]. Numerical simulations have also explored the effects of rotation on thermal convection and provided significant contributions [13]. Here, we consider both rotating and non-rotating convection and provide insights into heat transport scaling of rotating convective turbulence. Recent experimental work has elucidated the dependence of heat transport on Prandtl number as well as on Rayleigh and Rossby or Taylor numbers [5]. The velocity and vorticity fields [7], and related the crossover to rotation-dominated convection through an analysis in terms of Ekman boundary layers has also been done [10].

The main governing non dimensional parameters for this problem are the Rayleigh number, $R a$, which is the ratio of buoyancy forces to the product of momentum and thermal diffusivity and proportional to the vertical temperature difference, the Taylor number, $T a$, which is the ratio of the Coriolis force to viscous forces and proportional to the square of the rotation rate, and Prandtl number, $\mathrm{Pr}$, which is the ratio of thermal diffusivity to dynamics viscosity.

$$
R a=\frac{g \alpha \Delta T h^{3}}{v \kappa} \quad T a=\left(\frac{2 \Omega h^{2}}{v}\right)^{2} \quad \operatorname{Pr}=\frac{v}{\kappa}
$$

where $g$ is the gravitational force, $\alpha$ is the thermal expansion coefficient, $\Delta T$ is the temperature gradient, $h$ is the length scale, $v$ is the kinematic viscosity, $\kappa$ is the thermal diffusivity, and $\Omega$ is the rotation rate. The Rayleigh number in rotating flows can be compared to its critical value according to the theory of Chandrasekhar [1] as follows:

$$
R a=8.7 T a^{2 / 3}
$$

Rotation has a surprisingly small impact on heat transport when the flow is turbulent with a $20-30 \%$ enhancement under rotation caused by rotation-induced interactions in the convective boundary layer. This region has been well studied 
[9], [14]. The geostrophic regime at moderate and lower $R a$ and the crossover to turbulent convection, however, are much less thoroughly investigated and are very relevant for geophysical applications. Scientifically, there are numerical predictions that heat transport in the interesting region below the transition to strong turbulence is governed by vertically coherent vortices that span the cell height. There is controversy surrounding these structures and their role in heat transport. The effects of rotation on convection, especially on heat transport, might be expected to be substantial given that rotation profoundly changes the nature of boundary layer instability and modifies the length scales over which motions occur. Whereas thermal plumes are formed in long sheets and are swept across the cell by mean flow, rotation spins up these plumes into intense vortical structures. Furthermore, rotation is known to shorten the linear length scale dramatically as rotation is increased [14]. Additional ingredients introduced by rotation are the Ekman pumping or suction imposed by the differential rotation of the boundary and the interior flow and the dynamical constraints imposed by the Taylor-Proudman theorem for strongly rotating flows. Despite these interesting factors, previous heat transport measurements have not been well understood. This communication is discusses some preliminary experimental results on heat transport modification and thermal convection in a rotating flows. The rest of the paper is organized as follows: the experimental set up and flow dynamics are outlined in section II, followed by results and discussion in section III. Finally the conclusions are given in section IV.

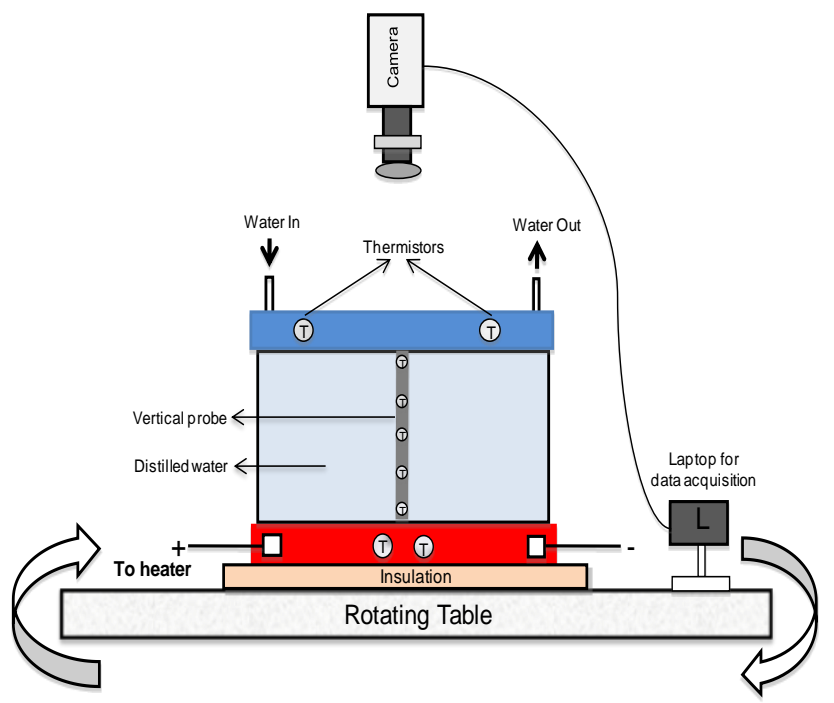

Fig. 1. Schematic of the experimental set up showing thermal convection in a rotating frame. The thermistors are embedded in the top and bottom plate and a vertical probe of thermistors is inserted into the fluid to measure the fluid heat transport.

\section{EXPERIMENTAL SET UP AND FLOW DYNAMICS}

The experimental facility is shown in Fig. 1. The experiments were conducted in a square tank with cross section (length, $L$ and width, $W$ ) $50.8 \mathrm{cmx} 50.8 \mathrm{~cm}$ and height $h=12.1 \mathrm{~cm}$. The tank was filled with distilled water for heat transport experiments, which was degassed to remove any air bubbles and impurities that might be present. This was important since the presence of bubbles might skew the measurements. The tank was placed on a rotating table (diameter, $d=36$ inches), which was supported by air bearings. The frequency of rotation, $f$, can be varied from $0-1$ $\mathrm{Hz}$, and was controlled using a variable frequency drive. The bottom plate was made up of Aluminum, side walls were made of Plexiglas for visualization and the top plate was made of Aluminum with an acrylic unit attached to it which aided in top cooling. In order to initiate turbulent convection, heat was provided from the bottom and the top part was cooled using a water feedback system. A film heater was attached to the bottom plate which provided constant heat flux, $Q$, to the fluid layer. The top plate was cooled by allowing water to flow through the slot channels present on the top plate. The flow of water was temperature controlled by a refrigerator circulator and then fed into the rotating frame through a water slip connection. The flow was further temperature regulated by a feedback temperature control unit that maintained the top-plate temperature constant with root-mean-square fluctuations of $\leq 1 \mathrm{mK}$. All electrical wires were fed into the rotating frame through an electrical slip ring and inside a hollowed steel shaft which also served as the drive train for rotation. The electrical noise of the slip ring was small enough that there was no measurable difference in the signals with or without rotation. The rotation frequency was measured accurately (to within $\pm 5 \%$ ) using a frequency counter. This ensured that the rotation rate was constant throughout the course of the experiment.

A total of eight thermistors were embedded in the bottom plate to measure the temperature. In addition four thermistors were embedded on the top plate to regulate the temperature of the top portion, thus providing a constant temperature gradient. The temperature gradient, $\Delta T$, drives the Convective Turbulence (CT) which causes heat transport and mixing inside the fluid layers. The bottom plate, side walls and the top plate were heavily insulated in order to avoid any losses to the ambient. In addition, the room temperature was maintained constant to minimize the effect of ambience on heat transfer measurements. A vertical probe consisting of six thermistors (shown in Figure 1) was held in contact with the fluid layer through a tiny port on the top plate. The thermistors were arranged in such a way that the temperature of the bottom, middle, and top fluid layer is obtained. These measurements will give accurate heat transport results. A video camera is installed for visualization purpose.

In pure conduction, heat is transported mainly due to molecular diffusion, however, for CT, heat can be advected by the fluid motion, thus transporting heat more efficiently [2]. The dimensional number that plays an important role in characterization of thermal convection is Nusselt number

$$
\mathrm{Nu}=\mathrm{Q}_{\text {convection }} / \mathrm{Q}_{\text {diffusion }}=\frac{\left(\dot{Q}-Q_{\text {loss }}\right) h}{k \Delta T}
$$

where $Q_{\text {loss }}$ is the sum of background losses due to the bottom and side insulations, and side wall heat loss, $k$ is the thermal conductivity. These losses are calculated and are included in the $\mathrm{Nu}$ measurements for accuracy [2], [10]. In addition to this, the losses due to the thickness of the Aluminum plate on bottom and top are also accounted in our measurements, since the thermistors are embedded into them and not directly 
inside the fluid. The convective heat transfer was thus obtained after subtraction of all the losses.

\section{RESULTS AND DISCUSSION}

The work by Chandrasekhar in 1953 [1], followed by others has shown that rotation due to the Coriolis parameter $(\Omega)$ modifies the problem of convective stability, by suppressing the onset of convection. The role of rotation on Rayleigh-Bénard convection is still largely unclear and the role of moderate $T a$ and $R a$ numbers on convective turbulence is not well understood. Thus in this study we will cover a range of Rayleigh number, $R a$, and Taylor number, $T a$, to study the onset of convection and turbulence. The list of the parameters used in our experiments is shown in Table 1. In our experiments we adopted the following methodology: the experiments were conducted for varying values of $R a$ for different rotation rates, effectively giving different $T a$. For e.g. for one particular rotation rate, a range of $R a$ was covered. Later the rotation rate was changed and the same range of $R a$ was covered.

TABLE I: PARAMETER RANGE FOR THIS STUDY

\begin{tabular}{lllll}
\hline \hline Run \# & Ta & Ra & $\mathrm{T}_{\mathrm{m}}$ & \multicolumn{1}{c}{$\mathrm{Ra}_{\mathrm{c}}$} \\
\hline 1 & 0 & $1 \times 10^{7}-2 \times 10^{8}$ & 23.2 & - \\
2 & $1.15 \times 10^{9}$ & $1 \times 10^{7}-2 \times 10^{8}$ & 23.2 & $0.9 \times 10^{7}$ \\
3 & $4.57 \times 10^{9}$ & $1 \times 10^{7}-2 \times 10^{8}$ & 23.2 & $2 \times 10^{7}$ \\
4 & $1.89 \times 10^{10}$ & $1 \times 10^{7}-2 \times 10^{8}$ & 23.2 & $3.5 \times 10^{7}$ \\
\hline \hline
\end{tabular}

This procedure was repeated for different sets of $T a$ shown in Table 1. In order to ensure that the $R a$ was same for different rotation rates, the mean cell temperature, $T_{m}$, was fixed, and the top and bottom temperatures were driven to a steady state using feedback loop systems. Thus, one could achieve results for different $T a$, for the same set of $R a$. As seen in Table 1, four sets of $T a$ were explored, namely, $T a=0$, $1.15 \times 10^{9}, 4.57 \times 10^{9}, 1.89 \times 10^{10}$. The range of $R a$ was $\approx$ $1 \times 10^{7}-2 \times 10^{8}$.

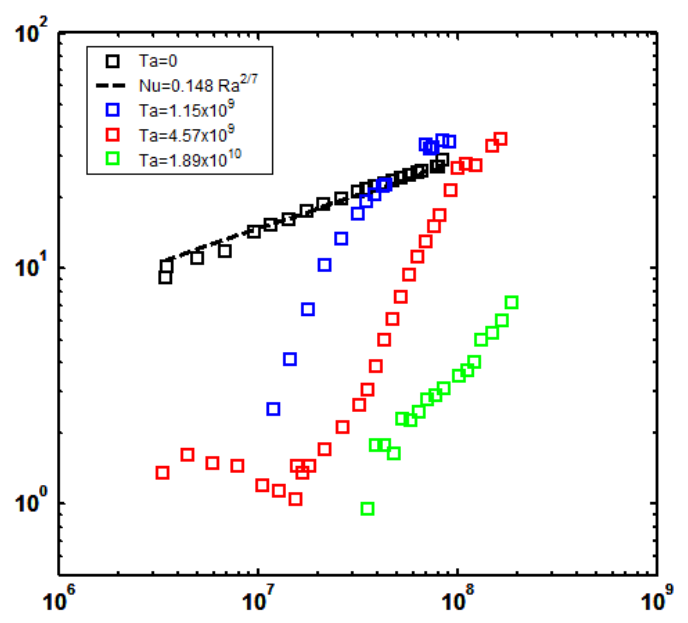

Fig. 2. Plot of convective heat transfer, Nu as a function of Ra for varying values of Ta. The dashed line is the empirical fit for the non-rotating case.

The heat transfer in thermal convective flows is characterized by the Nusselt number, $N u$. For the set of experimental runs shown in Table I, $N u$ was calculated using equation (2). The log-log plot of $N u$ as a function of $R a$ for varying values of rotation (Ta) is shown in Figure 2. For the case of no rotation $(T a=0)$, the $N u$ increases linearly with $R a$. This can be empirically modeled using the following formula

$$
N u=A R a^{\alpha}
$$

where $A=0.148$, and $\alpha=2 / 7$ for the present study. Similar parameterization have been proposed in previous studies [10], [15] with values of $A$ within $\pm 5 \%$, and $\alpha= \pm 2 \%$. For the case of moderate rotation, $T a=4.57 \times 10^{9}$, the first evident fact is that the $N u$ doesn't go below 1, which indicates the presence of wall mode. The point when $N u \approx 1$ is believed to be the beginning of the wall mode, where the convective structures are mostly present near the wall boundaries. For the case of $T a=4.57 \times 10^{9}$, after the wall mode, the $N u$ increases at a much faster rate than the case of no rotation. This is true for the other two rotation, namely low rotation rate $\left(T a=1.15 \times 10^{9}\right)$, and high rotation rate $\left(T a=1.89 \times 10^{10}\right)$ as well. The preliminary result indicates that the wall mode occurrence for the case of rotation occurs at $R a \approx 1-4 \times 10^{7}$ for all the three rotation rates. Beyond his value the bulk mode kicks in, where the convection is energetic in the fluid bulk. The bulk state can be modeled as $N u \approx R a^{2.2}$, which is very different from the non-rotating case. The $(N u-1)$ data was plotted as a function of the dimensional Rayleigh number $\varepsilon$, where $\varepsilon=\frac{R a}{R a_{c}}$. Here $R a$ is the measured Rayleigh number for our experiments, and $R a_{c}$ is the critical value of Rayleigh number corresponding to the crossover from wall mode to bulk thermal convection.

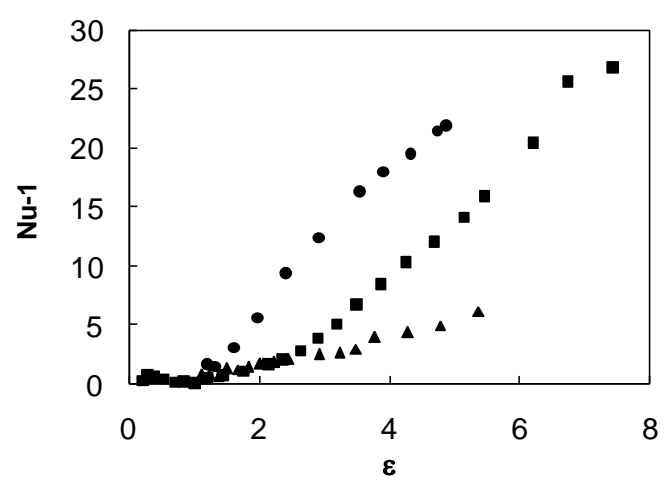

Fig. 3. Plot of Nusselt number as a function of.dimensional Rayleigh number. Symbols: circle $-\mathrm{Ta}=1.15 \times 10^{9}$, square $-\mathrm{Ta}=4.57 \times 10^{9}$, and triangle $\mathrm{Ta}=1.89 \times 10^{10}$.

The plot of $N u-1$ as a function of $\varepsilon$ is shown in Fig. 3. It can be seen from this figure that the side wall mode collapses for all three Ta, and the bulk mode follows a linear trend. The wall mode and the bulk mode can be fitted using two different fitting functions.

The vertical probe consisting of five thermistors arranged in the vertical $(z)$ direction gives the heat transport variation along the axis of rotation. In Fig. 4, the data from the five different thermistors are shown for the all the four $\mathrm{Ta}$ cases at the same Ra. The colors represent different thermistors (as stated in the figure caption). For the case of moderate rotation, one can see the presence of coherent structures in 
the vertical direction. These coherent structures are absent in the non-rotating case. This point to the fact that rotation enhances Ekman pumping and hence leads to Taylor-Proudman type vertical coherent structures which are aligned parallel to the rotation axis [12], [13]. These structures modify the heat transport and were also observed by Sakai [11]. The presence of these structures was confirmed in our experiments using the vertical probe measurements. It was also seen that the structures are much more stable at the low and moderate rotation.

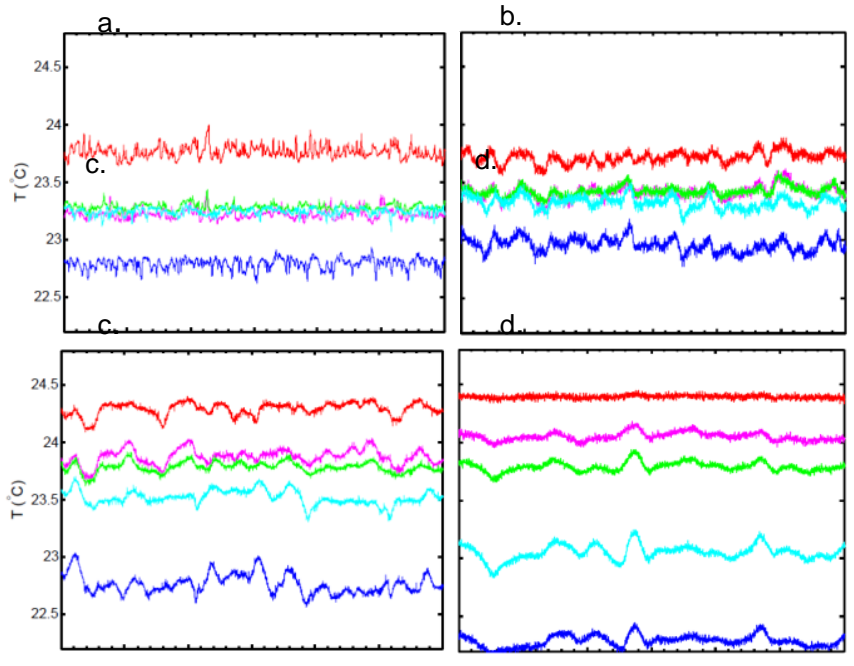

Fig. 4. Vertical probe measurements for four different $\mathrm{Ta}$ at $\mathrm{Ra}=7.98 \times 10^{7}$. The presence of coherent structures is evident in (b), (c) and (d). colors coding is as follows: red - T1 (close to the bottom plate), green, blue and magenta - T2, T3 and T4 (moving up in the vertical direction), blue - T5 (close to the top plate)
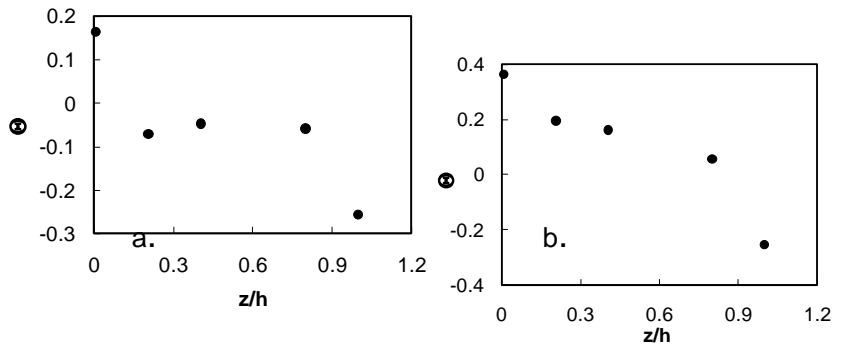

Fig. 5. Vertical heat transport measurements at varying $z / h$ for $\mathrm{Ta}=0$ (a) and $\mathrm{Ta}=4.57 \times 10^{9}(\mathrm{~b})$. The heat transport is more uniform for the case of rotation.

The vertical temperature gradient, $\Theta$ can be defined as

$$
\Theta=\frac{\overline{T_{V}}-T_{m}}{T_{b}-T_{T}}=\frac{\overline{T_{V}}-T_{m}}{\Delta T}
$$

where $T_{v}$ is the temperature of the vertical probe thermistors, $T_{m}$ is the mean cell temperature, $T_{b}$ is the bottom plate temperature, $T_{T}$ is the top plate temperature, and $\Delta T$ is the temperature gradient which is the temperature difference between the bottom and top plate. The plot of $\Theta$ is shown in Figure 5 for two different rotation rates, $T a=0$ and $T a=4.5 \times 10^{9}$. The vertical temperature profile is very different for both these cases. For the case of no rotation, the middle fluid layer is flat, indicating the absence of any vertical transport. On the other hand for the case of rotation, the vertical temperature gradient has a slope indicating thermal transport in the vertical direction. This crudely indicates the presence of vertical structures carrying heat in the direction of rotation, thus replicating the Ekman pumping phenomena. More work needs to be done in future to understand the dynamics of these vertical structures and their role in heat transport and convective circulation.

\section{CONCLUSIONS}

An experimental study of Rayleigh-Bénard turbulent convection in rotating and non-rotating frame in a square cross section is presented. For the case of non-rotating convection, the Nusselt number, $\mathrm{Nu}$, was found to scale roughly as $R a^{2 / 7}$. The square cell was subjected to three different rotation rates characterized by the Taylor number, $T a=1.15 \times 10^{9}, 4.57 \times 10^{9}$ and $1.89 \times 10^{10}$. For the case of rotating convection the $N u$ scaling was found to be $R a^{2.2}$. It was also found that for the case of rotating convection the flow can be characterized into two modes, namely, wall mode and bulk mode. It was found that the wall mode can be collapsed for all the three $\mathrm{Ta}$, while the bulk mode growth followed different slopes. Heat transport measurement in rotating convection confirmed the findings by other researchers that rotation enhances thermal transport over a certain range of $R a$ and $T a$ for a fixed Prandtl number of $P r=6$ [9]-[12]. This enhancement can attributed to the Ekman pumping of the boundary layer by rotation-induced vortices that are aligned parallel to the axis of rotation. The presence of the coherent structures was quantitatively confirmed.

\section{ACKNOWLEDGMENTS}

The first author, Sridhar Balasubramanian (S.B), would like to thank Los Alamos National Laboratory for providing research support for this project. S.B would also like to thank the incumbent institution, IIT Bombay for providing monetary support for attending this conference.

\section{REFERENCES}

[1] S. Chandrasekhar, "The instability of a layer of fluid heated below and subject to Coriolis forces," in Proc. R. Soc. Lond. A, vol. 217, pp. 306-327, 1953.

[2] G. Ahlers, S. Grossmann, and D. Lohse, "Heat transfer and large scale dynamics in turbulent Rayleigh-Benard convection." Rev. Mod. Phys., vol. 81, pp. 503-537, 2009.

[3] H. T. Rossby, "A study of Benard convection with and without rotation," J. Fluid Mech., vol. 36, pp. 309-335, 1969.

[4] B. M. Boubnov and G. S. Golitsyn, "Experimental study of convective structures in rotating fluids." J. Fluid Mech. vol. 167, pp. 503-531, 1986.

[5] H. J. S. Fernando, D. L. Boyer, and R. R. Chen, "Effects of rotation on convective turbulence," J. Fluid Mech., vol. 228, pp. 513-547, 1991.

[6] Y. Liu and R. E. Ecke, "Heat transport scaling in turbulent Rayleigh-Benard convection: effects of rotation and Prandtl number," Phys. Rev. Lett, vol. 79, pp. 2257-2260, 1997.

[7] S. N. Dikarev, "The influence of rotation upon the structure of convection in a deep homogeneous fluid," Dokl. Akad. Nauk SSSR, vol. 273, pp. 718-720, 1983.

[8] R. E. Ecke and Y. M. Liu, "Traveling-wave and vortex states in rotating Rayleigh-Benard convection," Intl J. Engng Sci., vol. 36, pp. 1471-1480, 1998.

[9] P. Vorobieff and R. E. Ecke, "Turbulent rotating convection: an experimental study," J. Fluid Mech., vol. 458, pp. 191-218, 2002.

[10] Y. Liu and R. E. Ecke, "Heat transport measurements in turbulent rotating Rayleigh-Bénard convection," Phys. Rev. E, vol. 80, pp. 1-12, 2009.

[11] S. Sakai, "The horizontal scale of rotating convection in the geostrophic regime," J. Fluid Mech., vol. 333, pp. 85-95, 1997. 
[12] E. M. King and J. M. Aurnou, "Thermal evidence for Taylor columns in turbulent rotating Rayleigh-Benard convection," Phys. Rev. E., vol. 85, pp. 1-11, 2012.

[13] I. Grooms, K. Julien, J. B. Weiss, and E. Knobloch, "Model of convective Taylor columns in rotating Rayleigh-Benard convection," Phys. Rev. Lett., vol. 104, pp. 1-4, 2010.

[14] S. Weiss, R. J. Stevens, J. Q. Zhong, H. J. Clercx, D. Lohse, and G. Ahlers, "Finite-Size Effects Lead to Supercritical Bifurcations in Turbulent Rotating Rayleigh-Bénard Convection," Phys. Rev. Lett., vol. 105 , pp. 1-4, 2010

[15] A. Nikolaenko and G. Ahlers, "Nusselt Number Measurements for Turbulent Rayleigh-Benard Convection," Phys Rev. Lett., vol. 91, pp. $1-4,2003$.

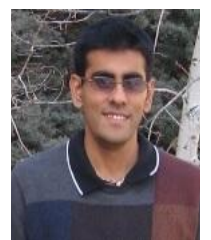

S. Balasubramanian obtained his B.E. in Mechanical Engineering from Shanmuga College of Engineering, India. $\mathrm{He}$ received his M.S and Ph.D. degrees in Mechanical Engineering from Arizona State University, USA. He is an AsSISTANT PROFESSOR in the Department of Mechanical Engineering at Indian Institute of
Technology, Bombay (IIT B). Prior to this he was a Postdoctoral RESEARCHER at Los Alamos National Laboratory (LANL). He has published over 15 journal papers and has numerous conference proceedings to his name.

Prof. Balasubramanian is a member of American Physics Society (APS) and American Institute of Aeronautics and Astronautics (AIAA). He is the recipient of Young Faculty Award at IIT Bombay and Los Alamos Awardees program at LANL.

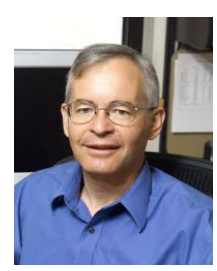

R. E. Ecke received his B.S. and Ph.D. degrees in Physics from University of Washington, USA. He is presently a TeChNICAL StAFF and DiRECTOR of Center for Nonlinear studies at Los Alamos National Laboratory. He has over hundred journal publications in the field of thermal convection research problem. Dr. Ecke is a member of American Physics Society (APS). $\mathrm{He}$ is the recipient of numerous prestigious awards such as Director's Fellow, Los Alamos Awardees program etc. His contribution in this field is remarkable. 